\title{
Ueber die Entmischung von kolloiden Lösungen.
}

Von Wolfgang Ostwald (Leipzig).

In einer früheren Arbeit) hat der Verfasser als ein besonders charakteristisches Kennzeichen der emulsoiden Beschaffenheit von kolloiden Systemen wie Gelatine-, Seifen- usw. Solen ihre Entmischbarkeit zu zwei zusammenhängenden Flïssigkeitsschichten hervorgehoben. Zwecks hesonderer Betonung dieser Eigentümlichkeit bat der. Verfasser an die Forscher, die in bezug auf diese Charakteristik der genannten Kolloide aicht ganz seiner Meinung waren, die Aufforderung gerichtet, $z$. B. ein Gold- oder Sulfidsol in zwei analog beschaffene Flüssigkeitsschichten koagulieren $z \mathfrak{u}$ wollen ", offenbar in der Meinung, dab ein solcher Versuch nicht glücken würde. In einer kürzlich erschienenen interessanten Arbeit von R. Zs ig mon dy ${ }^{2}$ ) ist, nun auf diese Aufforderung zurückgegriffen worden. Die Arbeit behandelt in der Tat in ihrem ersten Teil "die Entmischung verschiedener Kolloidlösungen, insbesondere von Arsensulfid und kolloiden Goldlösungen in zwei zusammenhängende, tropfbare Flüssigkeitsschichten," und es wird in ihr gezeigt, dab sich "typische ,Suspensionskolloide wie Arsensulfid, wie ,Emulsionskolloide in zwei homogene Flüssigkeitsschichten scheiden lassen “.

Es erscheint nach diesen Resultaten $R$. Zsigmondy's, als wenn die anfangs erwähnte Schlubfolgerung des Verfassers völlig irrtïmlich wäre und eine Berechtigung ganz und gar nicht vorläge, aus der Entmischbarkeit z. B. einer Seifenlösung in zwei Flüssigkeitsschichten beim Aussalzen, auf eine emulsoide Beschaffenheit des Ausgangssystems zu schlieBen. Der Verfasser bittet kurz zeigen zu dürfen, daß die Sachlage eine umgekehrte ist, insofern als der Irrtum in der Schlubfolgerung $R$. Zsigmondy's liegt.

R. Zsigm ondy erhält seine zwei Flüssigkeitsschichten dadurch, dab er zu seinen kolloiden Ausgangssystemen Alkohol und Aether hinzusetzt. Er arbeitet mit anderen Worten in einem System, dessen Dispersionsmittel ternär ist. Nun ist selbstverständlich bei der Charakteristik des Verfassers keinerlei Vorbehalt gemacht worden iber Art, Anzahl usw, der angewandten Koagulatoren. Wohl aber wurde dabei die Voraussetzung gemacht, $d$ a $d$ as Kolloid, die disperse Phase, selbst

1) Wo. Ostwald, Koll.-Zeitschr. 11, 230 (1912).

2) R. Zs ig nu ond y, Koll.-Zeitschr. 13, 105 (1913). wesentlich an dem Vorgang beteiligt ist, derart, daß in Abwesenheit desselben der betrachtete Vorgang, die Entmischung, nicht eintritt. Der Verfasser glaubt in der Tat nur solche Vorgänge ais charakteristisch für . kolloide Systeme ansehen zu sollen, in denen das Kolloid die makgebende Rolle spielt. Gewiß gibt es viele Erscheinungen, bei denen die Gegenwart eines Kolloids nur eine vorwiegend quantitative Aenderung irgendwclcher Konstanten reranlaBt; man denke an Diffusionen in Gallerten, an die Aenderung der Lösungsmittelkonslanten usw. Solche Vorgänge können aber offenbar nicht herangezogen werden, wenn es sich um qualitative Charakteristika erster Ordnung handelt, die für eine ganze grobe Klasse kolloider Zustände charakteristisch sein sollen.

Bei den Versuchen von R. Zsigmondy entstehen nun durch den sukzessiven Zusatz von Alkohol und Aether z. B. zu Arsensulfidsol zwei Flizssigkeitsschichten, eine obere ätherisehe und eine untere wasseralkoholische, die, falls Gleichgewichteingetreten ist, allein sämtliches Arsentrisulfid enthäl. Analoge Versuche gelingen beim Gold usw. R. Zsigm ondy betont, dak "beide Schichten vollkommen tropfbar flüsşig sind“. Der Verfasser erlaubt sich nicht, hieran zu zweifeln. Wohl aber" vermag er mit dem besten Willen nicht einzusehen, in welcher Beziehung diese gewib an und für sich interessanten Versuche zu den fraglichen kolloiden Entmischungsvorgängen stehen. Die Zsigmondy'sche Entmischung tritt ganz genausoein, wenn man statt des suspensoiden Kolloids eine verdünnte Kochsalzlösung, eine grobe Suspension oder aber reines Wasser nimmt. R. Zsig. m o n dy bemerkt selbst, daß z. B. "dem Arsen. sulfid eine mehr passive Rolle zuzukommen scheint ". Es handelt sich in der Tat um eine Entmischung des Dispersions mittels nicht aber des Kolloids, die dem Verfasser fundamental verschieden zu sein scheint von der Entmischung einer typischen emulsoiden Lösung zu zwei anders konzentrierten Kolloidlösungen, wie sie in der zitierten Arbeit aufgeführt wurden. Als zwei noch nicht erwähnte Beispiele seien noch $\beta$-Gelatine und Gummi arabicum genannt, die nach $O$. Büts chli bei Alkoholzusatz ebenfalls zwei derartige kolloide Flüssigkeitsschichten 
geben $^{3}$ ). Man versuche, analog den $\mathrm{Zsig}$ mondy'schen Versuchen die Gelatinelösung ohne Gelatine mit Alkohol zu zwei Flüssigkeitsschichten $\mathrm{zu}$ entmischen. Vielleicht zeigt dieser Versuch besonders deutlich, daB es sich bei den Zsigmondy'schen Entmischungserscheinungen um Vorgänge handelt, die in keiner wesentlichen Beziehung zu den besprochenen $\mathrm{k}$ oll o i d e n Entmischungsprozessen stehen. Der Verfasser weigert sich, Erscheinungen als charakteristisch für Kolloide anzusehen, bei denen das Kolloid tatsächlich "eine mehr passive Rolle" spielt.

Damit soll beileibe nicht etwa eine $A b$ lehnung der Zsigmondy'schen Versuche an und für sich ausgesprochen werden, falls eben von der besprochenen, dem Verfasser irrtïmlich erscheinenden Schlubfolgerung abgesehen wird. Es ist zweifellos von großem Interesse, den Einflub einer kolloiden Phase auf die Entmischung binärer und ternärer Dispersionsmittelgemenge zu untersuchen, speziell die Veränderungen der Löslichkeitskurven festzustellen, wie auch R. Zsigm ondy selbst bemerkt. Allerdings kann man von vornherein vermuten, $d a B$ auch diese Verhältnisse wenigstens von suspensoiden Kolloiden in ebenso geringfügigem Maße beeinfluBt werden wie andere Eigenschaften des Lösungsmittels, z. B. ihr Gefrier- und Siedepunkt ${ }^{4}$ ). Immerhin bedarf diese Folgerung noch der experimentellen Prüfung, wie sie von $R . Z$ sigmondy auch in Aussicht gestellt worden ist. Interessant ist weiterhin, dah von $R$. Zsigmondy ein einigermaßen stabiler Uebergang des Kolloids in die ätherische Phase nicht beobachtet werden konnte. Es wäre ja an und für sich gar nicht ausgeschlossen, daB auf diese Weise Aetherosole

9) O. Batsch 11, Untersuchungen über Strukturen (Lelpzig 1898) 54. - "Gegen Alkohol zeigte sie (die Gelatinelosung) ein nicht uninteressantes Verhalten, entsprechend dem im vorigen Abschnith fiir Gummi geschilderten. Wurde das zwei- bis dreifache Volum 40-50 proz. Alkohols zugesetzt, so tribte sie sich, wurde jedoch beim Schitteln wieder ganz klar; als dann tropfenweise absoluter Alkohol zugefügt wurde, so trat, nachdem eine ziemliche Quantität Alkohol zugegeben war, dauernde Trübung ein. Die Untersuchung ergab, daß diese Tribung von Flüssigkeitstropichen herrühre, die sich ziemlich rasch zu Boden setzten und sich über Nacht zu einer bräunlichen Flüssigkeit vereinigt hatten, welche etwa $1 / 3$ des Volumens der ursprïnglichen Gelatinelösung betrug."

1) Zwei vom Verfasser angestellte Handversuche in MeBzylindern iber die Entmischung eines Goldsols bei Alkoholatherzusatz ergaben $\mathrm{k}$ in e die Messungsfehler uberschreitenden Differenzen zu den entsprechenden Versuchen mit reinem Wasser. usw. entständen, analog den Ueberführungsversuchen Graham's von Hydrogelen in Alkogele usw.

Im zweiten Teil seiner Arbeit erörtert $R$. $Z$ sigm ondy einige allgemeinere Fragen uber die zweckmäBigste Systematik der kolloid. chemischen Phänomene. Der Verfasser legt besonderen Wert darauf, hervorzuheben, daß er ganz im allgemeinen eine erhebliche $\mathrm{An}$ näherung zwischen den Ansichten R. Zsigmo ndy's und seinen eigenen glaubt feststellen zu können. Diese erfreuliche Näherung beruht insbesondere auf der gemeinsamen Erkenntnis, daß zwei verschiedene Gesichtspunkte vorliegen, von denen aus zwei verschiedene systematische Prinzipien sich ergeben. Der eine Gesichtspunkt ist derjenige, welcher die speziellen kolloiden Eigentümlichkeiten der einzelnen Elemente und Verbindungen, die Kolloid. chemie der chemischen Spezies, in den Vordergrund der Betrachtung stellt; dies ist der Zsigmondy'sche Standpunkt. Der andere Gesichtspunkt, derjenige des Verfassers, geht aus von den physikalisch-chemischen Eigentümlichkeiten des kolloiden $Z u$ standes und seinen GeseizmäBigkeiten, und abstrahiert hierbei von den Spezialitäten der einzelnen Kolloidbeispiele. Es ist sicher, dab diese Verschiedenheit des Gesichtspunktes wesentlich zu den aufgetretenen Meinungsverschiedenheiten beigetragen hat, und dab durch die gemeinschaftliche Klarstellung dieses Punktes ein Teil dieser Differenzen sich von selbst behebt. Der Verfasser muß zwar gestehen, daß er in gewissen Einzelheiten den letzten Ausführungen R. Zs ig mondy's noch immer nicht völlig zustimmen kann. Indessen glaubt er sich auch darin mit R. Zsig m on dy einig, daB alle Systematik schlieBlich eine $Z$ weckmäBigkeitsfrage ist, deren Entscheidung am besten der selbstregulatorischen Entwicklung der Wissenschaft überlassen wird.

\section{Zus immenfassung.}

Die von R: Zsigmondy studierten Ent. mischungsvorgänge in Hydrosol - Alkohol - AetherGemischen haben nichts zu tun mit den Entmischungsvorgängen typischer emulsoider Systeme zu zwei Flüssigkeitsschichten (Aussalzen bei Seifenlösungen, Schichtenbildung in Gelatinelösungen bei Alkohol- und Neutralsalzzusatz), da erstere Vorgäıge in qualitativ gleicher Weise auch in molekularen Lösungen, groben Dispersionen und insbesondere auch im reinen Dispersionsmittel, d. h. in Ab- 
wesenheit des Kolloids vor sich gehen. Es handelt sich also um gewöhnliche Entmischungserscheinungen des ternären $D$ ispersionsmittels, nicht um für Kolloide irgendwie charakteristische Erscheinungen. Die Tatsache, daB auch typische Suspensoide wie Arsentri- sulfid- und Goldsole mit Alkohol und Aether zwei Flüssigkeitsschichten geben, kann daher nicht als im Widerspruch aufgefabt werden zu der Kennzeichnung der erwähnten tropfigen Entmischung als Charakteristikum typisch emulsoider Systeme.

\section{Literatur - Uebersicht.}

$\mathrm{SB} .=$ Sitzungsbericht. $\mathrm{R} .=$ Referat.

\section{Allgemeine Kapillarphysik.}

Bragg, W. H., Die Reflexion von Rontgenstrahlen an Kristallen. (Physikal. Zeitschr. 14, 472.)

- X-Strahlen und Kristalle. (Nature 90, 219, 1912.)

de Brogli e, M., Ueber die Zerstreuung und Reflexion der Röntgenstrahlen. (Compt. rend. 156, 1753.)

- u. F. A. Lindemann, Ueber die optischen Erscheinunger beim Auftreffen von Röntgenstrahlen auf kristalline Medien. (Compt. rend. 156, 1461.)

$\mathrm{C}_{\mathrm{b}}$ a u di e r, J., Ueber die Aenderung des magnetischen Rotationsvermögen bei Zustandsanderungen. (Compt. rend. 156, 1529.)

Détrait, R., Ueber das Gleiten von Fliussigkeiten an Wandungen. (Compt. rend. 156, 1670.)

Devaux, H., Recherches sur les lames d'huile étendues sur l'eau. (Tours 1912, 80,23 S.)

E w a Id , P.P., Zur Theorle der Interferenzen der Rontgenstrahlen in Kristallen. (Physik. Zeitschr. 14, 465.)

Goldsch midt, V. M., Ueber die innere Struktur der Kristalle. SB. Polyteknisk Forenings Kemikergruppe. Kristiania, 8. April 1913. (Chem.-Ztg. 1913, 992.)

$\mathrm{H}$ a g e $\mathrm{n}$ b a ch, A., Elektrischer Widerstand von Seifenhäutchen. (Arch.Sc.phys. et nat. Genève [4] 35, 329.)

$\mathrm{Herweg}, J$., Ueber die Beugungserscheinungen der Röntgenstrahlen am Gips. (Physik. Zeitschr. 14,417.)

Hirs ch, F., Uleber die Bildungsgesetze der Phosphoreszenzzentren bei den Erdalkaliphosphoren. (Diss., Heideiberg 1912, 69 S.)

Joly, J., u. E. R uth erf ord, Das Alter pleochroitischer Hófe. (Philos. Magazlne [6] 25, 644.)

L a ue, $M$., Kritische Benerkungen zu den Deutungen der Photogramme van Friedrich u. Knipping. (Physik. Zeitschr. 14, 421.)

$M$ a nde 1 sta $m$, L., Ueber die Rauhigkeit freier Flüssigkeitsoberflächen. (Ann. d. Phys. [4], 41, 609.)

Roberts, J. H.T., Die Desintegration der Metalle bei hohen Temperaturen. Kondensationskerne von heißen Drăhten. (Phil. Mag. [6] 25, 270, 1913.)

Sorkau, W., Ueber den Eirflue von Temperatur, spezifischem Gewicht und chemischer Konstitution auf Turbulenz-Reibung. (Diss., Greifswald 1912, $8^{0}, 110 \mathrm{~S}$.)

\section{Allgemeine Kapillarchemie.}

Befthoud, A. Theorie der Bildung von KristallAlachen. (Joum. de Chim. phys. 10, 624, 1912.)

Bleyer, B., u. S. W. Kaufmann, Ueber die Natur der alkalischen Berylliumlösurgen. (Zeitschr. f. anorg. Chern. 82, 71.)

Bruni, $G_{\text {, }}$ a. G. Scarpa, Studien zur Elektrolyse ktistallinischer Verbindungen. 1. Silberjodid. (Atti R. Accad. dei Lincei, Rom [5] 22 I, 438.)

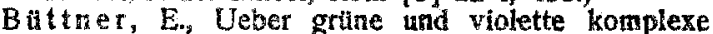
Chromiazetate. (Diss., Tubingen 1912, 80, 102 S.)
Chapman, D. L., Ein Beitrag zur Theorie der Eiektrokapillaritatt. (Philos. Magazine [6] 25, 475.)

Eder, R., Ueber die Mikrosublimation von Alkaloiden Im luftverdannten Raume. (Schweiz. Wchschr. f. Chem. u. Pharm. 51, 228, 241, 253.)

Fischer, Wold., Ueber temperaturreversible $\mathrm{Ab}$ sorptionsstreifen. R. 20. Hauptversammlung der Deutschen Bunsen-Gesellschaft für angewandte physikalische Chemie zu Breslau vom 3. bis 6. Aug. 1913. (Chem.-Ztg. 1913, 976.)

- W. M., Die Manganosulfide, ihre Darstellung, gegenseitigen Beziehungen und ihr analytisches Verhalten. SB. Chemische Gesellschaft am Rigaschen Polytechnischen Institut, 15. Februar 1913. (Chem-Ztg. 1913, 937).

Gaudefroy, $C_{\text {, }}$ Ueber die Entwässerungsfiguren. (Compt. rend. 156, 1387.)

- Ueber die Deshydratationsfiguren. SB. Académie des Sciences, Patis, 5. Mai 1913. (Chem.-Ztg. 1913, 847.)

Girard, P., La Pression osmotique et le Mécanisme de l'osmose. (A. Hermann et Fils, Paris 1912.)

Georgievics, G. v.. Ueber das Wesen des Vorgangs, welcher bei der Verteilung eines Stoffs zwischen zwel flüssigen Losungsmitteln stattfindet. (Zeitschr. f. physik. Chem. 84, 353, 1913.)

Grinakowsky, K., Ueber die Bildung von Kombinationsstreifung, Kombinationsebenen und Zerstörung von Chromalaunkristallen beim Auflósen. (Journ. d. russ. phys.- chem. Ges. 44, Chem. Teil, 802, 1912.)

Gutbier, A., H. Gebhardt u. B. Ottenstein, Ueber das Verhalten von Wasserstoff gegen Palladium. (Ber. d. Deutsch. chem. Ges. 46, 1453.)

Hantzsch, A, Ueber die Natur der gelben und roten Helianthinlösungen und die Chromoisomerie det Aminoazosalze. (Ber.d.Deutsch. chem. Ges.46,1537.)

Hey d w elller, A., Ueber physikalische Eigenschaften von Lósungen in ihrem Zusammenhang. IV. Refraktion, Dispersion und Dissoziation von Salzen im Wasser. (Ann. d. Phys. [4], 41, 499.)

Kue Bnet, H., Ueber Löslichkeitsunterschiede an Kristalloberflächen. (Zeitschr. f. physik. Chem. 84, $313,1913$.

Lehmann, $O$., Neue Untersuchungen über flüssige Kristalle. III.' (SB. der Heidelberger Akad. d. Wissenschaften. Matherm.-natarw. Klasse 1913, Abhdlg. 13.)

- Erforschung der Molethularkräfte durch Störung des molekularen Gleichgewichtes bei flüssigen Kristallen. (Verh. d. Deutsch. physik. Ges. 15, Nr. 10, 413, 1913.)

Pfeiffer, P., U. J. v. Modelski, Verhalten der $x$-Aminosäuren und Polypeptide gegen Neutralsalze. 11. Mitteilung. (Zeitschr. f. physiol. Chem. 85, 1.)

Piccard, J., Ueber Farben zweiter Ordnung und über holo- und merichinoide Salze. (Ber. d. Deutsch. chem. Ges. 46, 1843.y 